\title{
NAFLD determined by Dallas Steatosis Index is associated with poor outcomes in COVID-19 pneumonia: a cohort study
}

\author{
Paulina Moctezuma-Velázquez ${ }^{1}$. Godolfino Miranda-Zazueta ${ }^{1}$ - Edgar Ortiz-Brizuela ${ }^{3}$. Juan Alberto Garay-Mora ${ }^{4}$. \\ María Fernanda González-Lara ${ }^{3} \cdot$ Karla Maria Tamez-Torres ${ }^{3} \cdot$ Carla Marina Román-Montes $^{3}$. \\ Bruno Alejandro Díaz-Mejía ${ }^{5}$ - Esteban Pérez-García ${ }^{3} \cdot$ Marco Villanueva-Reza $^{3}$ - Monica Chapa-lbargüengoitia ${ }^{4}$. \\ Luis Uscanga-Domínguez ${ }^{2}$. José Sifuentes-Osornio ${ }^{5}$ - Alfredo Ponce-de-León ${ }^{3}$ - David Kershenobich-Stalnikowitz ${ }^{2}$. \\ Blanca Mota-Ayala ${ }^{2} \cdot$ Carlos Moctezuma-Velázquez ${ }^{2,6}$ (1)
}

Received: 11 August 2021 / Accepted: 15 January 2022 / Published online: 9 February 2022

(c) The Author(s), under exclusive licence to Società Italiana di Medicina Interna (SIMI) 2022

\begin{abstract}
Coronavirus disease 2019 is a worldwide health challenge. Liver steatosis diagnosis based on imaging studies has been implicated in poor outcomes of COVID-19 pneumonia, but results are inconsistent. The Dallas Steatosis Index (DSI) is an available calculator developed to identify patients with non-alcoholic fatty liver disease (NAFLD). We hypothesized that it would be associated with in-hospital mortality, intensive care unit admission (ICU), and invasive mechanical ventilation (IMV). We conducted a retrospective cohort study on inpatients with confirmed COVID-19 pneumonia between February 26 and April 11, 2020. We computed the DSI on admission, and patients with high DSI were considered with NAFLD. We employed logistic regression to study the association between NAFLD, mortality, ICU admission, and IMV. We studied the association between liver steatosis on computed tomography (CT) and these outcomes, and also between Metabolic Associated Fatty Liver Disease (MAFLD) based on CT findings and risk factors and the outcomes. 470 patients were included; 359 had NAFLD according to the DSI. They had a higher frequency of type 2 diabetes ( $31 \%$ vs $14 \%, p<0.001)$, obesity (58\% vs $14 \%, p<0.001)$, and arterial hypertension ( $34 \%$ vs $22 \%, p=0.02)$. In univariable analysis, NAFLD was associated with mortality, ICU admission, and IMV. Liver steatosis by CT and MAFLD were not associated with any of these outcomes. In multivariable logistic regression, high DSI remained significantly associated with IMV and death. High DSI, which can be easily computed on admission, was associated with IMV and death, and its use to better stratify the prognosis of these patients should be explored. On the other hand, liver steatosis by CT and MAFLD were not associated with poor outcomes.
\end{abstract}

Keywords COVID-19 $\cdot$ NAFLD $\cdot$ Liver steatosis $\cdot$ Fatty liver disease $\cdot$ Coronavirus

Carlos Moctezuma-Velázquez

mocmocte@hotmail.com

1 Department of Surgery, Instituto Nacional de Ciencias Médicas y Nutrición Salvador Zubirán, Vasco de Quiroga 15, Belisario Domínguez Sección 16, Tlalpan, 14080 Mexico City, Mexico

2 Department of Gastroenterology, Instituto Nacional de Ciencias Médicas y Nutrición Salvador Zubirán, Vasco de Quiroga 15, Belisario Domínguez Sección 16, Tlalpan, 14080 Mexico City, Mexico

3 Department of Infectious Diseases, Instituto Nacional de Ciencias Médicas y Nutrición Salvador Zubirán, Vasco de
Quiroga 15, Belisario Domínguez Sección 16, Tlalpan, 14080 Mexico City, Mexico

4 Department of Radiology, Instituto Nacional de Ciencias Médicas y Nutrición Salvador Zubirán, Vasco de Quiroga 15, Belisario Domínguez Sección 16, Tlalpan, 14080 Mexico City, Mexico

5 Department of Medicine, Instituto Nacional de Ciencias Médicas y Nutrición Salvador Zubirán, Vasco de Quiroga 15, Belisario Domínguez Sección 16, Tlalpan, 14080 Mexico City, Mexico

6 Division of Gastroenterology, Liver Unit, University of Alberta, Edmonton, AB, Canada 


\section{Introduction}

By the end of 2019, an outbreak of pneumonia of an unknown cause was identified in Wuhan, the capital of Hubei province, China. A novel coronavirus, later designated severe acute respiratory syndrome coronavirus 2 (SARS-CoV-2), was identified as its aetiological agent, and this condition was named coronavirus disease 2019 (COVID-19) [1]. As of June 21, 2021, COVID-19 has been confirmed in 178,118,597 patients and has caused 3,864,180 deaths around the world [2]. Using data from China, the overall case-fatality ratio (CFR) of COVID-19 has been estimated at around $1.38 \%$ (95\% confidence interval [CI] 1.23-1.53) [3]. Nonetheless, the probability of survival is largely influenced by several factors, such as age, sex, and the presence of some comorbidities [4].

Several comorbidities have been related to a worse prognosis of COVID-19, including a history of cardiovascular disease, diabetes, chronic respiratory disease, hypertension, cancer, and obesity [5-7]. The role of liver steatosis detected on imaging studies has been controversial: Zheng et al. [8] found steatosis to be a marker of a more severe disease course, as well as others [9-12], while Campos-Murguia et al. [13] found that it was liver fibrosis, assessed by the non-alcoholic fatty liver disease (NAFLD) Fibrosis Score, rather than steatosis, the one associated with the need for invasive mechanical ventilation (IMV) and mortality [13, 14]. In opposition to this, Castro et al. [15] failed to stablish an association between fibrosis and COVID-19 outcomes; in this work, steatosis was determined by the Hepatic Steatosis Index, and liver fibrosis was assessed by the AST to platelet ratio index, the NAFLD Fibrosis Score, and the Fiborisis-4 index. Therefore, more studies exploring the role of steatosis on the prognosis of COVID-19 beyond its association with disease severity are needed. The Dallas Steatosis Index (DSI) is a clinical tool that uses clinical variables and laboratory test results to identify patients at high risk of NAFLD; it has the advantage of not requiring imaging and therefore is readily available. The aim of this study was to determine the association between the DSI and poor COVID-19 outcomes: in-hospital mortality, ICU, and IMV. Secondary aims were to evaluate the association between liver steatosis determined by computed tomography (CT), and these outcomes, and also between Metabolic Associated Fatty Liver Disease (MAFLD) diagnosis based on CT and risk factors, and the outcomes of interest.

\section{Materials and methods}

\section{Study design, patients, and SARS-CoV-2 testing}

We conducted a retrospective cohort study in a tertiary care center in Mexico City dedicated to the care of adults with severe and critical COVID-19 based on the US Coronavirus Disease 2019 (COVID-19) Treatment Guidelines illness categories [16]. We included 470 consecutive subjects with SARS-CoV-2 infection confirmed by real-time reverse transcription-polymerase chain reaction (RT-PCR) that were admitted between February 26 and April 11, 2020. COVID19 diagnosis was based on the definitions described by the World Health Organization. Inpatients were followed until discharge or death (last status update was on May 15th, 2020). SARS-CoV-2 testing was carried out on nasopharyngeal or oropharyngeal swabs. Nucleic acid extraction was performed using the NucliSens EasyMAG system ${ }^{\circledR}$ (bioMérieux, Boxtel, The Netherlands). RT-PCR was carried out on an Applied Biosystems 7500 thermocycler (Applied Biosystems, Foster City, CA, USA) using primers and conditions described elsewhere [17]. The study was reviewed and approved by the Institutional Review Board (ref. no. 3333). Written informed consent was waived because of the observational nature of the study.

\section{Data collection}

We extracted clinical and epidemiological data at presentation using a standardized case report form [18]. The rest of the information was obtained from electronic clinical records. Imaging and laboratory studies were performed according to our institutional recommendations. Suggested basal assessment includes complete blood cell count, a comprehensive metabolic panel, inflammatory markers (i.e., C-reactive protein, ferritin, and procalcitonin), creatine kinase, fibrinogen, D-dimer, and lactate dehydrogenase.

\section{Assessment of non-alcoholic fatty liver disease with the Dallas Steatosis Index}

The DSI is a calculator to identify patients at high risk of NAFLD, and it was developed using logistic regression, considering NAFLD as liver steatosis detected by magnetic resonance spectroscopy in the absence of secondary causes of liver steatosis, including significant alcohol intake. The DSI has a C-statistic of 0.824 [19], and it takes into account age, ALT, triglycerides, body mass index, glucose, ethnicity, sex, type 2 diabetes, and arterial hypertension. The DSI was computed with an online calculator according to the original formula (https://dsi.wustl.edu). According to the original publication, patients with a DSI $\geq 0$ are considered at high risk of NAFLD, and therefore, for this study, all patients with DIS $\geq 0$ were considered to have NAFLD. The logistic regression equation is the following:

DSILOGIT $=-9.4+0.316$ (if age $\geq 50$ and female $)+2.4$ (if known diabetes) $+0.02 \times$ (equals 0 if diabetic; if not diabetic equals the glucose concentration in $\mathrm{mg} / \mathrm{dl}$ ) +0.3 (if known hypertension) +0.5 (if Hispanic/Asian/Other race/ 
ethnicity $+\mathrm{Ln}$ triglycerides in $\mathrm{mg} / \mathrm{dl}+0.4$ if ALT 13.5 $-19.49 \mathrm{IU} / \mathrm{L}+1.1$ (if ALT $19.5-40 \mathrm{IU} / \mathrm{L}+1.5$ if ALT $>40$ $\mathrm{IU} / \mathrm{L}+0.7$ if not black and BMI $25-27.49 \mathrm{~kg} / \mathrm{m}^{2}$ and +1.4 if not black and BMI $27.5-34.9 \mathrm{~kg} / \mathrm{m}^{2}+1.9$ if not black and $35-37.49 \mathrm{~kg} / \mathrm{m}^{2}+2.6$ if not black and $>37.5 \mathrm{~kg} / \mathrm{m}^{2}-0.2$ if black and BMI $25-27.49 \mathrm{~kg} / \mathrm{m}^{2}$ and +0.8 if black and BMI $27.5-34.9 \mathrm{~kg} / \mathrm{m}^{2}+0.8$ if black and $35-37.49 \mathrm{~kg} / \mathrm{m}^{2}+1.8$ if black and $>37.5 \mathrm{~kg} / \mathrm{m}^{2}$.

\section{Assessment of liver steatosis with computed tomography and diagnosis of metabolic-associated fatty liver disease}

A computed tomography (CT) of the chest is suggested in our institution for all hospitalized patients before admission. The diagnosis of liver steatosis was made by an experienced radiologist (JAGM) blinded to patient outcomes. For this purpose, we analyzed six liver (four in the right lobe and two in the left lobe) and two spleen regions of interest (ROI) in non-contrast $\mathrm{CT}$ scans $(1.0 \pm 0.2 \mathrm{~cm})$ per patient. Attenuation measures were collected from each ROI, and the mean value in Hounsfield Units (HU) was obtained for each tissue and used to compute the liver/spleen ratio. The cut-off value to consider liver steatosis was liver/spleen ratio $<1$ [20]. MAFLD was diagnosed when a patient with steatosis on CT had any of the following three conditions: (1) BMI $\geq 25 \mathrm{~kg} / \mathrm{m}^{2}$; (2) diabetes mellitus; (3) two or more of the following risk factors: triglycerides $\geq 150 \mathrm{mg} / \mathrm{dl}$; arterial hypertension; prediabetes.

\section{Statistical analysis}

Categorical variables were reported as frequencies and proportions. Continuous variables were described using median and interquartile range (IQR). Variables between patients with and without NAFLD as per the DSI were compared with the chi-squared test and the Mann-Whitney $U$ test, as appropriate. Patients were followed until death and were censored at the time of discharge. We used univariable logistic regression to evaluate the association between the different variables and the outcomes of interest (i.e., inhospital death, ICU, IMV). Afterward, variables with a $p$ value of $\leq 0.1$ were included in multivariable analysis. A two-sided $p$ value of less than 0.05 was considered statistically significant. We used the Liu method of empirical point estimation to determine a suitable cut-off point of the DSI to identify MAFLD. All the analyses were performed using Stata 14 (StataCorp, Texas, USA). The study was reviewed and approved by the Institutional Review Board (ref. no. 3333). Written informed consent was waived because of the observational nature of the study.

\section{Results}

\section{General characteristics of patients}

We identified 470 patients with SARS-CoV-2 infection that were admitted during the study period. Two hundred ninetyeight $(63 \%)$ patients were male; the median age was 51 years (IQR 42-62). The prevalence of obesity, diabetes mellitus, and hypertension were $47 \%, 27 \%$, and $31 \%$, respectively. Three hundred and fifty-nine patients $(76.38 \%)$ were considered to have NAFLD according to the DSI. According to the CT analysis, 320 patients (70\%) had liver steatosis, and of them, 292 (92\%) had MAFLD. One hundred twenty-seven (27\%) patients died, 206 (44\%) required ICU admission, and $128(27 \%)$ IMV. The rest of the characteristics are shown in Table 1.

\section{Variables associated with NAFLD}

When compared to patients without NAFLD as per the DSI, patients with NAFLD had a higher body mass index [30.8 (IQR 28.3-34.7) vs 25.4 (IQR 23.7-27.4) $\left.\mathrm{kg} / \mathrm{m}^{2}, p<0.001\right]$, and a higher rate of obesity ( 58 vs $14 \%, p<0.001$ ), type 2 diabetes ( 31 vs $14 \%, p<0.001$ ), arterial hypertension (34 vs $22 \%, p<0.001$ ), and liver steatosis detected on CT (74 vs $55 \%, p<0.001)$. Patients with NAFLD as per the DSI had lower oxygen saturation levels at admission [84 (IQR 70-88) vs $86 \%$ (IQR 80-89), $p=0.002$ ], and higher D-dimer [727.5 (IQR 453-1136) vs 616 (IQR 391-1094) ng/mL $p=0.04$ ] and lactate dehydrogenase levels [399.5 (IQR 316-540) vs 347.5 (IQR 264-472) U/L, $p<0.001$ ], but also higher lymphocyte count [808.5 (IQR 589-1088) vs 737.3 (IQR 504-976) $\mu \mathrm{L}, p=0.03]$.

\section{Association between NAFLD and mortality}

There were 127 deaths: $21(19 \%)$ and $106(30 \%)$ in patients without and with NAFLD as per the DSI $(p=0.03)$. On univariable logistic regression analysis, features related with in-hospital mortality were age (OR $1.05,95 \%$ CI 1.04-1.07, $p<0.001$ ), sex (OR 1.94, 95\% CI 1.23-3.04, $p=0.004$ ), diabetes mellitus, (OR 1.78, 95\% CI 1.14-2.76, $p=0.01$ ), arterial hypertension (OR $1.64,95 \%$ CI 1.07-2.52, $p=0.02$ ), oxygen saturation on room air (OR $0.92,95 \% \mathrm{CI}$ $0.90-0.94, p<0.001$ ), total lymphocytes (OR $0.99,95 \%$ CI $0.997-0.999, p<0.001)$, triglyceride levels (OR 1.00, 95\% CI $1.00-1.004, p=0.004$ ), and NAFLD as per the DSI (OR $1.79,95 \%$ CI $1.06-3.04 p=0.03$ ). On multivariable analysis, NAFLD as per the DSI remained significantly associated with mortality (OR 2.13, 95\% CI 1.05-4.34, $p=0.04$ ) (Table 2). 
Table 1 General characteristics

\begin{tabular}{|c|c|c|c|c|}
\hline Variables & $N=470$ & No NAFLD $*, N=111$ & NAFLD*,$N=359$ & $p$ \\
\hline Male, $n(\%)$ & $298(63)$ & $67(60)$ & $231(64)$ & 0.4 \\
\hline Age years (IQR) & $51(42-62)$ & $52(42-66)$ & $51(42-61)$ & 0.1 \\
\hline BMI kg/m² (IQR) & $29.7(26.7-33.4)$ & $25.4(23.7-27.4)$ & $30.8(28.3-34.7)$ & $<0.001$ \\
\hline $\mathrm{BMI} \geq 25, n(\%)$ & $406(86)$ & $62(56)$ & $344(96)$ & $<0.001$ \\
\hline $\mathrm{BMI} \geq 30, n(\%)$ & $223(47)$ & $15(14)$ & $208(58)$ & $<0.001$ \\
\hline Type 2 diabetes mellitus, $n(\%)$ & $126(27)$ & $15(14)$ & $111(31)$ & $<0.001$ \\
\hline Arterial hypertension, $n(\%)$ & $147(31)$ & $25(22)$ & $122(34)$ & 0.02 \\
\hline Chronic kidney disease, $n(\%)$ & $12(2)$ & $5(4)$ & $7(2)$ & 0.1 \\
\hline Liver steatosis on CT, $n(\%)$ & $320(70)$ & $60(55)$ & $260(74)$ & $<0.001$ \\
\hline MAFLD**, $n(\%)$ & $294(92 \%)$ & $39(65 \%)$ & $255(98 \%)$ & $<0.001$ \\
\hline Heart rate (IQR) & $102.5(90-115)$ & $102(87-114)$ & $103(90-116)$ & 0.1 \\
\hline Respiratory rate (IQR) & $28(22-34)$ & $26(21-30)$ & $28(22-35)$ & 0.005 \\
\hline Oxygen saturation on room air \% (IQR) & $85(74-88)$ & $86(80-89)$ & $84(70-88)$ & 0.002 \\
\hline Mean arterial pressure mmHg (IQR) & $90.2(83.2-97.2)$ & $88.5(83.2-94.8)$ & $90.9(83.4-98.4)$ & 0.06 \\
\hline Glucose, mg/dl, (IQR) & $101.5(90-126)$ & $93(85-105)$ & $105(94-130)$ & $<0.001$ \\
\hline Creatinine mg/dl, (IQR) & $0.95(0.77-1.16)$ & $0.93(0.74-1.15)$ & $0.95(0.79-1.16)$ & 0.5 \\
\hline Total bilirubin mg/dl (IQR) & $0.59(0.46-0.79)$ & $0.55(0.44-0.78)$ & $0.6(0.46-0.80)$ & 0.1 \\
\hline ALT U/L (IQR) & $37.7(24.3-55.3)$ & $24.1(16.2-37.7)$ & $41.5(27-59.6)$ & $<0.001$ \\
\hline AST U/L (IQR) & $43.7(31.6-66)$ & $35(25.6-50)$ & $49.3(34-67.8)$ & $<0.001$ \\
\hline ALP U/L (IQR) & $87(70-115)$ & $84(68-110)$ & $89(70-117)$ & 0.4 \\
\hline Albumin mg/dl (IQR) & $3.7(3.3-4.0)$ & $3.7(3.4-4.03)$ & $3.7(3.3-4.0)$ & 0.4 \\
\hline Triglycerides, mg/dl (IQR) & $151(115-200)$ & $121(94-160)$ & $164(125-212)$ & $<0.001$ \\
\hline CRP mg/dl (IQR) & $15.4(8.2-22.6)$ & $14.6(7.2-21.0)$ & $16.3(8.9-23.5)$ & 0.06 \\
\hline Lymphocytes $\mu \mathrm{l}$ (IQR) & $792(558.9-1057.8)$ & 737.3 (504-976.5) & $\begin{array}{l}808.5 \\
(588.9-1087.5)\end{array}$ & 0.03 \\
\hline D-Dimer, ng/ml (IQR) & $700(442-1106)$ & $616(391-1094)$ & $727.5(453-1136)$ & 0.04 \\
\hline CPK mg/dl (IQR) & $119(65-243.5)$ & $107(57-244)$ & $125(70-243)$ & 0.1 \\
\hline LDH U/L (IQR) & $393(304.5-513)$ & $\begin{array}{l}347.5 \\
(263.5-472)\end{array}$ & $\begin{array}{l}399.5 \\
(316.5-539.5)\end{array}$ & $<0.001$ \\
\hline Ferritin $\mu \mathrm{g} / \mathrm{l}$ (IQR) & 666.5 (332-1089.7) & $\begin{array}{l}622.7 \\
(227.5-1104)\end{array}$ & $\begin{array}{l}689.4 \\
(353.7-1078.5)\end{array}$ & 0.1 \\
\hline
\end{tabular}

$A L P$ alkaline phosphatase, $A L T$ alanine aminotransferase, $A S T$ aspartate aminotransferase, $B M I$ body mass index, $C P K$ creatine phosphokinase, $C R P$ C-reactive protein, $C T$ computed tomography, $I Q R$ interquartile range, $L D H$ lactate dehydrogenase, MAFLD metabolic-associated fatty liver disease, $N A F L D$ non-alcoholic fatty liver disease

*NAFLD according to the DSI (i.e. $\geq 0$ )

**MAFLD based on steatosis on CT plus any of the following three conditions: (1) BMI $\geq 25 \mathrm{~kg} / \mathrm{m}^{2}$; (2) diabetes mellitus; (3) two or more of the following risk factors: triglycerides $\geq 150 \mathrm{mg} / \mathrm{dl}$; arterial hypertension; prediabetes

\section{Association between NAFLD and ICU}

Two hundred and six patients required ICU: 31 (28\%) and $175(49 \%)$ patients without and with NAFLD as per the DSI, respectively $(p<0.001)$. On univariable logistic regression analysis, NAFLD as per the DSI (OR 2.45, 95\% CI 1.54-3.90, $p<0.001$ ), age (OR 1.02, 95\% CI $1.00-1.03, p=0.008$ ), sex (OR 2.18, 95\% CI 1.47-2.23, $p<0.001$ ), oxygen saturation on room air (OR $0.90,95 \%$ CI $0.88-0.92, p<0.001$ ), total lymphocytes (OR $0.998,95 \%$ CI $0.998-0.999, p<0.001$ ), and triglyceride levels (OR 1.005, $95 \%$ CI $1.002-1.007,<0.001)$ were associated with ICU admission (Table 3). On multivariable analysis, NAFLD as per the DSI showed a tendency to be associated with ICU admission (OR 1.71, 95\% CI 0.95-3.06, $p=0.07$ ).

\section{Association between NAFLD and IMV}

One hundred and twenty-eight patients required IMV: 11 $(10 \%)$ and 117 (32\%) patients without and with NAFLD as per the DSI, respectively $(p<0.001)$. On univariate logistic regression analysis, NAFLD as per the DSI (OR $4.40,95 \%$ CI $2.26-8.51, p<0.001)$, sex (OR $1.60,95 \%$ CI 
Table 2 Factors associated with in-hospital mortality

\begin{tabular}{|c|c|c|c|c|c|c|}
\hline & \multicolumn{3}{|c|}{ Univariable } & \multicolumn{3}{|c|}{ Multivariable } \\
\hline & OR & $95 \% \mathrm{CI}$ & $p$ & OR & $95 \% \mathrm{CI}$ & $p$ \\
\hline Age & 1.05 & $1.04-1.07$ & $<0.001$ & 1.06 & $1.04-1.08$ & $<0.001$ \\
\hline Sex & 1.94 & $1.23-3.04$ & 0.004 & 1.69 & $0.96-2.97$ & 0.07 \\
\hline Obesity & 0.95 & $0.63-1.42$ & 0.8 & - & - & - \\
\hline DM & 1.78 & $1.14-2.76$ & 0.01 & 1.09 & $0.61-1.94$ & 0.8 \\
\hline Arterial hypertension & 1.64 & $1.07-2.52$ & 0.02 & 0.67 & $0.37-1.21$ & 0.2 \\
\hline Oxygen saturation on room air & 0.92 & $0.90-0.94$ & $<0.001$ & 0.93 & $0.91-0.94$ & $<0.001$ \\
\hline Total lymphocytes & 0.99 & $0.997-0.999$ & $<0.001$ & 0.998 & $0.998-0.999$ & 0.004 \\
\hline NAFLD* & 1.79 & $1.06-3.04$ & 0.03 & 2.13 & $1.05-4.34$ & 0.04 \\
\hline Liver steatosis on CT & 0.98 & $0.63-1.55$ & 0.9 & - & - & - \\
\hline Triglycerides & 1.00 & $1.00-1.004$ & 0.004 & 1.00 & $0.99-1.00$ & 0.5 \\
\hline MAFLD** & 0.66 & $0.28-1.53$ & 0.3 & - & - & - \\
\hline
\end{tabular}

$C I$ confidence interval, $C T$ computed tomography, DM type 2 diabetes mellitus, MAFLD metabolic-associated fatty liver disease, $N A F L D$ non-alcoholic fatty liver disease, $O R$ odds ratio

*NAFLD according to the DSI (i.e. $\geq 0$ )

**MAFLD based on steatosis on CT plus any of the following three conditions: (1) BMI $\geq 25 \mathrm{~kg} / \mathrm{m}^{2}$; (2) diabetes mellitus; (3) two or more of the following risk factors: triglycerides $\geq 150 \mathrm{mg} / \mathrm{dl}$; arterial hypertension; prediabetes

\begin{tabular}{|c|c|c|c|c|c|c|}
\hline & \multicolumn{3}{|c|}{ Univariable } & \multicolumn{3}{|c|}{ Multivariable } \\
\hline & OR & $95 \% \mathrm{CI}$ & - & OR & $95 \% \mathrm{CI}$ & $p$ \\
\hline Age & 1.02 & $1.00-1.03$ & 0.008 & 1.01 & $0.99-1.03$ & 0.2 \\
\hline Sex & 2.18 & $1.47-2.23$ & $<0.001$ & 2.14 & $1.31-3.49$ & 0.002 \\
\hline Obesity & 1.16 & $0.80-1.66$ & 0.4 & - & - & - \\
\hline $\mathrm{DM}$ & 1.40 & $0.93-2.11$ & 0.1 & 0.82 & $0.48-1.38$ & 0.4 \\
\hline Arterial hypertension & 0.98 & $0.66-1.45$ & 0.9 & - & - & - \\
\hline Oxygen saturation on room air & 0.90 & $0.88-0.92$ & $<0.001$ & 0.91 & $0.89-0.93$ & $<0.001$ \\
\hline Total lymphocytes & 0.998 & $0.998-0.999$ & $<0.001$ & 0.999 & $0.998-0.999$ & 0.02 \\
\hline NAFLD* & 2.45 & $1.54-3.90$ & $<0.001$ & 1.71 & $0.95-3.06$ & 0.07 \\
\hline Liver steatosis on CT & 1.11 & $0.74-1.67$ & 0.6 & - & - & - \\
\hline Triglycerides & 1.005 & $1.002-1.007$ & $<0.001$ & 1.003 & $1.00-1.006$ & 0.01 \\
\hline MAFLD** & 1.86 & $0.78-4.41$ & 0.2 & - & - & - \\
\hline
\end{tabular}

$C I$ confidence interval, $C T$ computed tomography, DM type 2 diabetes mellitus, $M A F L D$ metabolic-associated fatty liver disease, NAFLD non-alcoholic fatty liver disease, $O R$ odds ratio

*NAFLD according to the DSI (i.e. $\geq 0$ )

**MAFLD based on steatosis on CT plus any of the following three conditions: (1) BMI $\geq 25 \mathrm{~kg} / \mathrm{m}^{2}$; (2) diabetes mellitus; (3) two or more of the following risk factors: triglycerides $\geq 150 \mathrm{mg} / \mathrm{dl}$; arterial hypertension; prediabetes
$1.03-2.49, p=0.03$ ), obesity (OR $1.56,95 \%$ CI $1.03-2.34$, $p=0.03$ ), oxygen saturation on room air (OR $0.96,95 \% \mathrm{CI}$ $0.94-0.97, p<0.001$ ), and triglyceride levels (OR 1.006, 95\% CI 1.003-1.008, $p<0.001)$ were associated with IMV. On multivariable analysis, NAFLD as per the DSI remained statistically significantly associated with IMV (OR 2.50, 95\% CI 1.20-5.21, $p=0.01$ ) (Table 4).

\section{Association between liver steatosis by $\mathrm{CT}$ and outcomes of interest}

There was no association between liver steatosis evaluated by CT and mortality (OR $0.98,95 \%$ CI $0.63-1.55, p=0.9$ ), ICU admission (OR 1.11, 95\% CI $0.74-1.67, p=0.6$ ), or IMV (OR 1.30, 95\% CI 0.81-2.06, $p=0.3$ ). 
Table 4 Factors associated with invasive mechanical ventilation need

\begin{tabular}{|c|c|c|c|c|c|c|}
\hline & \multicolumn{3}{|c|}{ Univariable } & \multicolumn{3}{|c|}{ Multivariable } \\
\hline & OR & $95 \% \mathrm{CI}$ & $\mathrm{p}$ & OR & $95 \% \mathrm{CI}$ & $\mathrm{P}$ \\
\hline Age & 0.98 & $0.97-1.00$ & 0.1 & 0.98 & $0.96-0.99$ & 0.01 \\
\hline Sex & 1.60 & $1.03-2.49$ & 0.03 & 1.33 & $0.81-2.21$ & 0.3 \\
\hline Obesity & 1.56 & $1.03-2.34$ & 0.03 & 1.04 & $0.63-1.72$ & 0.9 \\
\hline $\mathrm{DM}$ & 1.28 & $0.82-2.01$ & 0.3 & - & - & - \\
\hline Arterial hypertension & 0.73 & $0.46-1.15$ & 0.2 & - & - & - \\
\hline Oxygen saturation on room air & 0.96 & $0.94-0.97$ & $<0.001$ & 0.97 & $0.95-0.98$ & $<0.001$ \\
\hline Total lymphocytes & 0.999 & $0.998-1.00$ & 0.07 & 0.99 & $0.99-1.00$ & 0.1 \\
\hline NAFLD* & 4.40 & $2.26-8.51$ & $<0.001$ & 2.50 & $1.20-5.21$ & 0.01 \\
\hline Liver steatosis on CT & 1.30 & $0.81-2.06$ & 0.3 & - & - & - \\
\hline Triglycerides & 1.006 & $1.003-1.008$ & $<0.001$ & 1.004 & $1.001-1.006$ & $<0.001$ \\
\hline MAFLD** & 3.22 & $0.94-11.01$ & 0.06 & - & - & - \\
\hline
\end{tabular}

$C I$ confidence interval, $C T$ computed tomography, $D M$ type 2 diabetes mellitus, MAFLD metabolic-associated fatty liver disease, $N A F L D$ non-alcoholic fatty liver disease, $O R$ odds ratio

*NAFLD according to the DSI (i.e. $\geq 0$ )

**MAFLD based on steatosis on CT plus any of the following three conditions: (1) BMI $\geq 25 \mathrm{~kg} / \mathrm{m}^{2}$; (2) diabetes mellitus; (3) two or more of the following risk factors: triglycerides $\geq 150 \mathrm{mg} / \mathrm{dl}$; arterial hypertension; prediabetes

\section{Association between MAFLD and outcomes of interest}

Two hundred ninety-two (92\%) patients out of 320 who had steatosis on CT had MAFLD. There was no association between MAFLD and mortality (OR 0.66, 95\% CI $0.28-1.53, p=0.3$ ), ICU admission (OR $1.86,95 \%$ CI $0.78-4.41, p=0.2$ ), or IMV (OR 3.22, 95\% CI 0.94-11.01, $p=0.06)$.

\section{Performance of the DSI to diagnose MAFLD}

The DSI had sensitivity, specificity, positive predictive value, negative predictive value, and an area under the curve of $86.7 \%, 80.8 \%, 98.1 \%, 35 \%$, and 0.840 , respectively, for the diagnosis of MAFLD. We used the Liu method for empirical cut point estimation and found DSI $>0.16$ as a suitable cut-off point to identify MAFLD, with sensitivity, specificity, and area under the curve of $84 \%, 92 \%$, and 0.880 , respectively. Based on this new cut-off point, 342 (73\%) patients would have MAFLD in our sample.

\section{Discussion}

Metabolic syndrome and its components are associated with worse outcomes in patients with COVID-19 [7]. Nevertheless, the role of NAFLD on COVID-19 prognosis, recently redefined as "metabolic associated fatty liver disease" (MAFLD) [21], has only been partially evaluated, perhaps because of the indolent course of steatosis, the poor awareness of the general population about this condition, and limitations inherent to how NAFLD/MAFLD is diagnosed. In this study, we assessed the relationship between NAFLD diagnosed by the DSI and different COVID-19 outcomes, and we found NAFLD as per the DSI to be associated with death and IMV need in hospitalized patients with COVID-19.

Our findings can be explained by the pathophysiological link between preexisting chronic liver diseases like liver steatosis, exacerbated by COVID-19. Both assaults (liver steatosis and COVID-19) lead to an amplified pro-inflammatory state with high levels of inflammatory cytokines and, consequently, poor outcomes [22-24]. Unfortunately, we did not measure cytokine levels, which could help clarify this association. Moreover, cholangiocytes and hepatocytes express angiotensin-converting enzyme 2, and SARS CoV-2 could be acting as a second hit in the presence of liver steatosis to trigger a pro-inflammatory state [25]. Importantly, obesity based on the BMI was not associated with the outcomes of interest after adjusting by NAFLD as per the DSI, suggesting that it is fat distribution more than a patient's weight what matters, as it is visceral obesity the pro-inflammatory one.

The role of NAFLD in COVID-19 has been explored previously, but this is the first study using the DSI score, which is more accurate for diagnosing NAFLD when compared to other methods and risk tools [19]. In particular, CT has poor sensitivity for the diagnosis of mild steatosis, whereas the DSI was developed using magnetic resonance spectroscopy, which has high sensitivity even for mild steatosis [26]. Two meta-analyses about liver steatosis and COVID-19 concluded that NAFLD is associated with a severe disease course and ICU admission, but not with 
mortality [27]. These results are partly following our findings where patients with NAFLD as per the DSI had an OR 2.50 for IMV in multivariate analysis. In a study by Zheng et al. [8] in 66 patients with MAFLD, obesity was associated with increased COVID-19 severity; however, since all patients had MAFLD determined by CT and the criteria described in 2020 [21], its prognostic role in terms of outcomes of interest could not be assessed. A case-control study evaluating the severity of the disease rather than the outcomes [12] was able to link MAFLD defined by CT scan, with severe COVID-19; the same results were confirmed by other authors $[10,11,28]$.

Part of the intrinsic difficulties of studying NAFLD in these patients includes how to define this entity. For example, some studies have relied exclusively on imaging, which has some limitations, the first of them being that ultrasound and CT have poor sensitivity for mild steatosis. Also, even if it is very probable that most liver steatosis found on CT is NAFLD, strictly speaking, secondary causes need to be excluded, and also, it should be taken into account that COVID-19 itself may induce liver steatosis [28]. In this fashion, Ibañez et al. [29] found no association between MAFLD and poor outcomes, though MAFLD was defined exclusively based on CT findings and not on the recently proposed definition of MAFLD [21]. In this same study, the authors described an association between fibrosis and IMV need, but the authors assessed fibrosis with the FIB-4 index, which takes into account AST and ALT levels, which can be elevated due to different reasons during the disease course. It also has to be taken into account that criteria for ICU admission or initiation of IMV vary across centers and may be subject to availability, which may in part explain the inconsistency in the results between the different studies.

To the best of our knowledge, this is the first study assessing NAFLD by the DSI and COVID-19 outcomes, and it is also one of the few studies reporting the association between NAFLD and mortality. The DSI, unlike other methods, does not require imaging studies and was developed to diagnose NAFLD per se, including cases with mild steatosis. At this point, we cannot know if it is NAFLD that is associated with death and IMV or the combination of variables included in the DSI, many of which are well-known risk factors for poor outcomes in this population. However, even after adjusting for triglyceride levels, DM, obesity, arterial hypertension, and age, the DSI was still associated with poor outcomes. Of note, triglyceride levels were also associated with IMV and with ICU admission, and more recently have caught attention as a potential prognostic marker in patients with COVID-19. The intense reaction induced in COVID-19 ends up decreasing the activity of the lipoprotein lipase resulting in high triglyceride levels, which may be a valuable marker of the inflammatory state [30].
We acknowledge that our study does have limitations. First, the DSI takes into account glucose and triglycerides which in some patients were probably not taken in a fasting state, and AST and ALT levels, which may be elevated in COVID-19 due to other reasons. However, being our results in consonance with those found in other studies supports that these patients did have NAFLD, and that NAFLD is associated with poor outcomes. Also, our study was restricted to inpatients, and therefore, we could not evaluate the role of NAFLD in outpatients. Then, the prevalence of NAFLD as per the DSI that we found in our study is one of the highest reported in the literature so far and raises concern about some sort of overdiagnosis; however, this could be primarily due to two reasons: first, there is a baseline high prevalence of NAFLD in our population (i.e., Hispanics); and second, our study was conducted in patients with severe COVID-19, who are known to have a higher prevalence of comorbid conditions, including metabolic and cardiovascular diseases, when compared to non-severe cases. For example, the prevalence of DM in our study was $27 \%$ compared to the $6 \%$ reported by Wang et al. [31] Kreling et al. [32] also found a higher prevalence of liver steatosis, diagnosed by CT scan, among patients with COVID-19 when compared with negative controls. Finally, we did not have information available about each patient's usual alcohol intake, and therefore, it may be possible for some cases of steatosis to be alcoholrelated, which is the reason why we decided to stick to the term "steatosis on CT" instead of "NAFLD by CT", and also because the COVID-19 itself has been associated with histopathological findings of steatosis [28].

In conclusion, NAFLD as per the DSI is associated with the need for IMV and death in patients with severe COVID-19, and its use to better stratify the prognosis of these patients should be explored.

\section{Declarations}

Conflict of interest The authors declare no conflict of interests and we have no disclosures.

Human and animal rights statement The study was reviewed and approved by the Institutional Review Board (ref. no. 3333).

Informed consent Written informed consent was waived because of the observational nature of the study.

\section{References}

1. Wang D, Hu B, Hu C, Zhu F, Liu X, Zhang J et al (2020) Clinical characteristics of 138 hospitalized patients with 2019 Novel coronavirus-infected pneumonia in Wuhan, China. JAMA [Internet]. 323(11):1061-1069. https://doi.org/10.1001/jama.2020.1585 
2. World Health Organization. Situation Reports. https://www.who. int/emergencies/diseases/novel-coronavirus-2019/situation-repor ts/. Accessed Jun 2021

3. Verity R, Okell LC, Dorigatti I, Winskill P, Whittaker C, Imai N et al (2020) Estimates of the severity of coronavirus disease 2019: a model-based analysis. Lancet Infect Dis [Internet]. 20(6):669677. https://doi.org/10.1016/S1473-3099(20)30243-7

4. Ruan S (2020) Likelihood of survival of coronavirus disease 2019. Lancet Infect Dis [Internet]. 20(6):630-631. https://doi.org/10. 1016/S1473-3099(20)30257-7

5. Kass DA, Duggal P, Cingolani O (2020) Obesity could shift severe COVID-19 disease to younger ages. Lancet (London, England). 395:1544-1545

6. Lighter J, Phillips M, Hochman S, Sterling S, Johnson D, Francois $\mathrm{F}$ et al (2020) Obesity in patients younger than 60 years is a risk factor for COVID-19 hospital admission. Clin Infect Dis 71:896-897

7. Wu Z, McGoogan JM (2020) Characteristics of and important lessons from the coronavirus disease 2019 (COVID-19) Outbreak in China: summary of a report of 72314 cases from the Chinese Center for Disease Control and Prevention. JAMA [Internet]. 323(13):1239-1242. https://doi.org/10.1001/jama.2020.2648

8. Zheng KI, Gao F, Wang XB, Sun QF, Pan KH, Wang TY et al (2020) Letter to the Editor: Obesity as a risk factor for greater severity of COVID-19 in patients with metabolic associated fatty liver disease. Metabolism 108:154244

9. Ji D, Qin E, Xu J, Zhang D, Cheng G, Wang Y, Lau G (2020) Non-alcoholic fatty liver diseases in patients with COVID-19: A retrospective study. J Hepatol 73(2):451-453. https://doi.org/10. 1016/j.jhep.2020.03.044

10. Targher G, Mantovani A, Byrne CD, Wang XB, Yan HD, Sun QF et al (2020) Risk of severe illness from COVID-19 in patients with metabolic dysfunction-associated fatty liver disease and increased fibrosis scores. Gut 69(8):1545-1547

11. Roca-Fernández A, Dennis A, Nicholls R, McGonigle J, Kelly M, Banerjee R et al (2021) Hepatic steatosis, rather than underlying obesity, increases the risk of infection and hospitalization for COVID-19. Front Med 8(March):1-8

12. Gao F, Zheng KI, Wang XB, Yan HD, Sun QF, Pan KH et al (2021) Metabolic associated fatty liver disease increases coronavirus disease 2019 disease severity in nondiabetic patients. J Gastroenterol Hepatol 36(1):204-207

13. Campos-Murguía A, Román-Calleja BM, Toledo-Coronado IV, González-Regueiro JA, Solís-Ortega AA, Kúsulas-Delint D et al (2021) Liver fibrosis in patients with metabolic associated fatty liver disease is a risk factor for adverse outcomes in COVID-19. Dig Liver Dis 53(5):525-533

14. Belda González I, Soliva Martínez D, Castro García A, Cantero Ayllon MJ, de Miguel IR (2017) Gastric neumatosis: when not to operate. Cir Esp 6:121-123

15. Lopez-mendez I, Aquino-matus J, Gall SM, Prieto-nava JD, Juarez-hernandez E, Uribe M et al. (2020) Since January 2020 Elsevier has created a COVID-19 resource centre with free information in English and Mandarin on the novel coronavirus COVID-19. The COVID-19 resource centre is hosted on Elsevier Connect, the company's public news and information

16. Health NI of Coronavirus Disease 2019 (COVID-19) Treatment Guidelines (2020) Disponible en: https://covid19treatmentguid elines.nih.gov/. Vol. 2019, Nih

17. Corman VM, Landt O, Kaiser M, Molenkamp R, Meijer A, Chu DKW et al (2020) Detection of 2019 novel coronavirus (2019$\mathrm{nCoV}$ ) by real-time RT-PCR. Eurosurveillance 25(3):1-8
18. Dirección General de Epidemiología. Lineamiento Estandarizado para la Vigilancia Epidemiológica y por Laboratorio de Enfermedad por 2019-nCOV. https://www.gob.mx/salud/documentos/ 79208. Accessed Apr 2020

19. McHenry S, Park Y, Browning JD, Sayuk G, Davidson NO (2020) Dallas steatosis index identifies patients with nonalcoholic fatty liver disease. Clin Gastroenterol Hepatol [Internet]. 18(9):20732080.e7

20. Kerut SE, Balart JT, Kerut EK, McMullan MR (2017) Diagnosis of fatty liver by computed tomography coronary artery calcium score. Echocardiography 34(6):937-938

21. Eslam M, Sanyal AJ, George J (2020) MAFLD: a consensusdriven proposed nomenclature for metabolic associated fatty liver disease. Gastroenterology 158(7):1999-2014.e1

22. Li Y, Xiao SY (2020) Hepatic involvement in COVID-19 patients: pathology, pathogenesis, and clinical implications. J Med Virol 92(9):1491-1494

23. Hui JM, Hodge A, Farrell GC, Kench JG, Kriketos A, George J (2004) Beyond insulin resistance in NASH: TNF-alpha or adiponectin? Hepatology 40(1):46-54

24. Mehta P, McAuley DF, Brown M, Sanchez E, Tattersall RS, Manson JJ (2020) COVID-19: consider cytokine storm syndromes and immunosuppression. Lancet [Internet]. 395(10229):1033-1034. https://doi.org/10.1016/S0140-6736(20)30628-0

25. Hamming I, Timens W, Bulthuis MLC, Lely AT, Navis GJ, van Goor H (2004) Tissue distribution of ACE2 protein, the functional receptor for SARS coronavirus. A first step in understanding SARS pathogenesis. J Pathol 203(2):631-637

26. Lee S, Kim JH, Kim SY, Byun JH, Kim HJ, Kim M-H et al (2018) Comparison of diagnostic performance between CT and MRI in differentiating non-diffuse-type autoimmune pancreatitis from pancreatic ductal adenocarcinoma. Eur Radiol 28(12):5267-5274

27. Singh A, Hussain S, Antony B (2021) Non-alcoholic fatty liver disease and clinical outcomes in patients with COVID-19: a comprehensive systematic review and meta-analysis. Diabetes Metab Syndr 15(3):813-822

28. Díaz LA, Idalsoaga F, Cannistra M, Candia R, Cabrera D, Barrera F et al (2020) High prevalence of hepatic steatosis and vascular thrombosis in COVID-19: a systematic review and meta-analysis of autopsy data. World J Gastroenterol 26(48):7693-7706

29. Ibáñez-Samaniego L, Bighelli F, Usón C, Caravaca C, Carrillo CF, Romero $\mathrm{M}$ et al (2020) Elevation of liver fibrosis index FIB-4 is associated with poor clinical outcomes in patients with COVID19. J Infect Dis 222(5):726-733

30. Barman HA, Pala AS, Dogan O, Atıcı A, Yumuk MT, Alici G et al (2021) Prognostic significance of temporal changes of lipid profile in COVID-19 patients. Obes Med. 28:100373

31. Wang G, Wu S, Wu C, Zhang Q, Wu F, Yu B et al (2021) Association between non-alcoholic fatty liver disease with the susceptibility and outcome of COVID-19: a retrospective study. J Cell Mol Med 25:11212-11220

32. Medeiros AK, Barbisan CC, Cruz IR, de Araújo EM, Libânio BB, Albuquerque KS et al (2020) Higher frequency of hepatic steatosis at CT among COVID-19-positive patients. Abdom Radiol [Internet]. 45(9):2748-2754. https://doi.org/10.1007/ s00261-020-02648-7

Publisher's Note Springer Nature remains neutral with regard to jurisdictional claims in published maps and institutional affiliations. 